\title{
Guest editorial of the IJCARS MICCAI 2016 special issue
}

\author{
Sebastien Ourselin ${ }^{1,6}$ - Mert R. Sabuncu ${ }^{2}$ - William Wells ${ }^{7}$ - Leo Joskowicz ${ }^{3}$. \\ Gozde Unal $^{4}$ - Andreas Maier ${ }^{5}$
}

Published online: 25 July 2017

(C) CARS 2017

It is a pleasure to present this special issue of IJCARS that contains several of the best papers that were submitted to the 19th International Conference on Medical Image Computing and Computer-Assisted Intervention (MICCAI 2016) which was held in Athens, Greece. MICCAI 2016 was organized in a collaboration among University College London, Harvard Medical School, the Hebrew University of Jerusalem, and Bogazici, Sabanci, and Istanbul Technical Universities.

\footnotetext{
Andreas Maier

andreas.maier@fau.de

Sebastien Ourselin

s.ourselin@ucl.ac.uk

Mert R. Sabuncu

msabuncu@cornell.edu

William Wells

sw@bwh.harvard.edu

Leo Joskowicz

josko@cs.huji.ac.il

Gozde Unal

gozde.unal@itu.edu.tr

1 Centre for Medical Image Computing, University College London, London, UK

2 School of Electrical and Computer Engineering, Cornell University, 300 Rhodes Hall, Ithaca, NY 14853, USA

3 CASMIP Lab: Computer-Aided Surgery and Medical Image Processing Laboratory, The Hebrew University of Jerusalem, Jerusalem, Israel

4 Computer Engineering Department, Istanbul Technical University, Istanbul, Turkey

5 Computer Science, Friedrich-Alexander University Erlangen-Nuremberg Computer Science, Erlangen, Germany

6 Wellcome/EPSRC Centre for Interventional and Surgical Sciences, University College London, London, UK

7 Radiology, Harvard Medical School, Boston, MA, USA
}

For MICCAI 2016 there were a total of 756 submitted papers of which only 228 were accepted for presentation at the conference after a double-blind review process. The papers that scored best in this process got invitations to special issues in the International Journal of Computer Assisted Radiology and Surgery and Medical Image Analysis. Six of the 18 invited papers made it through the review process for this special issue which we proudly present in the following.

Sparks et al. propose an automated planning approach for the placement of intracranial electrodes in "Anatomydriven multiple trajectory planning (ADMTP) of intracranial electrodes for epilepsy surgery." The aim of the work is to reduce manual planning time from hours down to few minutes. Their approach was validated in 20 patients and could show that $70 \%$ percent of the electrodes could be planned automatically. In "Versatile, robust, and efficient tractography with constrained higher-order tensor fODFs" Ankele et al. show a novel approach for multi-fiber tractography that is fast and robust. Runtime could be reduced by a factor of approximately two, and condition numbers were lowered at least by one order of magnitude compared to other state-of-the-art methods. Zhang et al. propose a novel approach for appearance modeling in their paper "Deformable appearance pyramids for anatomy representation, landmark detection and pathology classification." Their approach proposes a multi-scale feature representation that is built from either Gaussian or wavelet pyramids. Results demonstrate improvements over the state of the art. In "An image retrieval framework for real-time endoscopic image retargeting" Ye et al. present an approach to support endoscopic gastrointestinal follow-up interventions. In order to do so, they present an image descriptor that is invariant to longtime tissue changes and a novel matching method for this descriptor. In experiments with 13 videos from six patients they show that their new approach is able to outperform the 
state of the art with respect to precision, recall, and computation time. Azizi et al. in "Detection and grading of prostate cancer using temporal enhanced ultrasound: combining deep neural networks and tissue mimicking simulations" propose to use temporal enhanced ultrasound to determine the malignancy of prostate lesions. Their approach is based on deep belief networks, and results indicate that there is a relation between micro-vibrations in micro-tissue and low-frequency spectral features found in temporal enhanced ultrasound. In "Consistent reconstruction of 4D fetal heart ultrasound images to cope with fetal motion" Tanner et al. propose to multi-sweep ultrasound to construct images that are consistent in space, phase, and time. In simulation and in vivo data the authors demonstrate that their approach outperforms the state of the art.

We believe that this special issue provides a representative selection from the various topics covered in MICCAI. We hope that the readers will enjoy this collection of papers. We would like to thank the reviewers of the papers and the staff in the editorial office of the International Journal of Computer Assisted Radiology and Surgery for their efforts and contributions. 